\title{
Coloring Chains for Compression with Uncertain Priors
}

\author{
Noah Golowich \\ Harvard University \\ Cambridge, MA, U.S.A. \\ ngolowich@college.harvard.edu
}

Submitted: Sep 18, 2016; Accepted: Sep 14, 2018; Published: Dec 21, 2018

(C) The author. Released under the CC BY-ND license (International 4.0).

\begin{abstract}
Haramaty and Sudan considered the problem of transmitting a message between two people, Alice and Bob, when Alice's and Bob's priors on the message are allowed to differ by at most a given factor. To find a deterministic compression scheme for this problem, they showed that it is sufficient to obtain an upper bound on the chromatic number of a graph, denoted $U(N, s, k)$ for parameters $N, s, k$, whose vertices are nested sequences of subsets and whose edges are between vertices that have similar sequences of sets. In turn, there is a close relationship between the problem of determining the chromatic number of $U(N, s, k)$ and a local graph coloring problem considered by Erdös et al. We generalize the results of Erdős et al. by finding bounds on the chromatic numbers of graphs $H$ and $G$ when there is a homomorphism $\phi: H \rightarrow G$ that satisfies a nice property. We then use these results to improve upper and lower bounds on $\chi(U(N, s, k))$.
\end{abstract}

Mathematics Subject Classifications: 05C15, 05C12

\section{Introduction}

We consider the following graph coloring problem. For a positive integer $N$, a chain of length $f$ and size $s$ is a nested sequence of sets $A_{0} \subseteq A_{1} \subseteq \cdots A_{f} \subseteq[N]$ with $\left|A_{0}\right|=1$ and $\left|A_{f}\right|=s$. We denote such a chain by $\left\langle A_{0}, A_{1}, \ldots, A_{f}\right\rangle$; if $\alpha$ is the single element of $A_{0}$, we will also write $\left\langle\alpha, A_{1}, \ldots, A_{f}\right\rangle$. For a chain $\mathcal{A}$, given by $\left\langle A_{0}, \ldots, A_{f}\right\rangle, S^{1}(\mathcal{A})$ is defined [5] to be the set of all chains $\left\langle B_{0}, \ldots, B_{f-1}\right\rangle$ such that for $0 \leqslant i \leqslant f-1, A_{i-1} \subseteq B_{i} \subseteq A_{i+1}$, where $A_{-1}=\varnothing$. Next, for a positive integer $j$ and a positive real number $x, \log ^{(j)} x$ denotes the base- 2 logarithm function iterated $j$ times. Moreover, $\log ^{*} x$ denotes the minimum $j$ such that $\log ^{(j)} x \leqslant 1$. Haramaty and Sudan [5] showed that for any $k, s \in \mathbb{N}$ the set of all chains of length $2 k$ and size at most $s$ can be colored with $2^{6(s+1)} \cdot \log ^{(k)} N$ colors so that for chains $\mathcal{A}, \mathcal{A}^{\prime}$ in this set with $S^{1}(\mathcal{A}) \cap S^{1}\left(\mathcal{A}^{\prime}\right) \neq \varnothing$ and $A_{0} \neq A_{0}^{\prime}, \mathcal{A}$ and 
$\mathcal{A}^{\prime}$ receive different colors. As we will show in Section 3, this result is equivalent to the fact that the following graph, which we denote by $U(N, s, k)$, has a proper coloring with $2^{6(s+1)} \cdot \log ^{(k)} N$ colors: $V(U(N, s, k))$ is the set of all chains of length $k$ and size at most $s$, and

$$
\begin{aligned}
E(U(N, s, k))= & \left\{\left(\left\langle\alpha, A_{1}, \ldots, A_{k}\right\rangle,\left\langle\beta, B_{1}, \ldots, B_{k}\right\rangle\right)\right. \\
& \left.: \alpha \neq \beta, \alpha \in B_{1}, \beta \in A_{1}, \quad \forall 1 \leqslant i \leqslant k-1: A_{i} \subseteq B_{i+1}, B_{i} \subseteq A_{i+1}\right\} .
\end{aligned}
$$

In this paper we prove an upper bound on the chromatic number of $U(N, s, k)$ that improves the upper bound found by Haramaty and Sudan when $s, k$ are small compared to $N$, and we also prove a lower bound on the chromatic number of $U(N, s, k)$ that greatly improves upon previous lower bounds. Before doing so, we introduce some notation and explain the motivation behind determining the chromatic number of the graphs $U(N, s, k)$.

Notation. We use the following conventions. For a chain $\mathcal{A}=\left\langle A_{0}, A_{1}, \ldots, A_{f}\right\rangle$, we let $s z(\mathcal{A})$ denote the size of $\mathcal{A}$, i.e. $s z(\mathcal{A})=\left|A_{f}\right|$. For $N \in \mathbb{N}$, we let $C h a i n(N)$ denote the set of all chains $\left\langle A_{0}, A_{1}, \ldots, A_{f}\right\rangle$ with $f \in \mathbb{N}$ and $A_{f} \subset[N]$. Given a graph $G$, we let $\chi(G)$ denote the chromatic number of $G, V(G)$ denote the set of vertices of $G, E(G)$ denote the set of edges of $G$, and for $v \in V(G), N(v)$ denote the set of neighbors of $v$ (excluding $v$ itself). All of our graphs have no loops; that is, for $(u, v) \in E(G), u \neq v$. For $\delta \geqslant 0$, we let $N_{\delta}(v)$ denote the set of all vertices of distance at most $\delta$ from $v$ (so that, for instance, $N(v) \cup\{v\}=N_{1}(v)$ ). For sets $S, T$ and a map $f: S \rightarrow T$, for a subset $H \subset S$, we let $f(H)=\{f(s): s \in H\}$. For a graph $G$ and a subset $T \subset V(G)$, we let $G[T]$ be the subgraph of $G$ induced by $T$. We use log to denote the logarithm base 2 and ln to denote the natural logarithm.

\subsection{Motivation}

The purpose in [5] of determining the chromatic number of the graphs described above was to solve the following compression problem: for some finite universe $U$, suppose that Alice is operating under the belief that a message $m$ is chosen from $U$ according to the probability distribution $P$, and that Bob operates under the belief that $m$ is chosen according to the distribution $Q$. Both Alice and Bob know that their distributions $P, Q$ are "close" in the sense that they know of some $\Delta \geqslant 0$ such that $\max _{m \in U}\left(\max \left(\log _{2} \frac{P(m)}{Q(m)}, \log _{2} \frac{Q(m)}{P(m)}\right)\right) \leqslant \Delta$. The smallest such $\Delta$ for which this inequality holds is denoted $\delta(P, Q)$. For $m$ drawn from $U$ according to $P$ (written as $m \sim_{P} U$ ), Alice wishes to communicate $m$ to Bob using a number of bits that is as small as possible in expectation.

Juba et al. [6] considered a version of this question when Alice and Bob are allowed to share common random bits, and showed that if so, they can communicate with $H(P)+$ $2 \Delta+O(1)$ bits. Haramaty and Sudan considered this problem when Alice and Bob are not allowed to share common random bits. To state their setup, we let $\mathcal{P}(U)$ be the space of probability distributions over $U$; an uncertain deterministic compression scheme is a pair of functions $E: \mathcal{P}(U) \times U \rightarrow\{0,1\}^{*} \cup\{\perp\}$ and $D: \mathcal{P}(U) \times\left(\{0,1\}^{*} \cup\{\perp\}\right) \rightarrow U \cup\{\perp\}$ such that for $P, Q \in \mathcal{P}(U)$ with $\delta(P, Q) \leqslant \Delta, \forall m \in U$, either $E(P, m)=\perp$ (which happens with some small probability) or $D(Q, E(P, m))=m$. In other words, with high 
probability $E(P, m) \neq \perp$, meaning that the encoding $E$ does not fail, and Bob can use the decoding function $D$ to recover the message $m$. Moreover, we want the expected length of the encoding $\mathbb{E}_{m \sim_{P} U}|E(P, m)|$ to be small. If the probability that $E(P, m)=\perp$ is 0 , then the compression scheme is said to have no error.

The following compression scheme was introduced in [5]: if we let $N=|U|$, then given $P \in \mathcal{P}(U), m \in U$, let $r=\left\lfloor-\log _{2} P(m)\right\rfloor$ and $f=2\left\lfloor\log ^{*} N\right\rfloor-1$. We now define a chain $\mathcal{A}$ of length $f$ by setting $A_{0}=\{m\}$ and for $1 \leqslant k \leqslant f, A_{k}=\left\{m^{\prime} \in[N]\right.$ : $\left.\left|\log _{2}\left(1 / P\left(m^{\prime}\right)\right)-r\right| \leqslant k \Delta+1\right\}$. Since $P, Q$ are $\Delta$-close, Bob knows that $-\log _{2} Q(m)$ is within $\Delta+1$ of $r$, and in general, for each $m^{\prime} \in A_{k}$ with $k \geqslant 1$, that $-\log _{2} Q\left(m^{\prime}\right)$ is within $(k+1) \Delta+1$ of $r$. Bob now constructs a chain $\mathcal{B}$, given by $\left\langle B_{0}, \ldots, B_{f-1}\right\rangle$, such that $B_{0}=\{w\}$ for some $w$ with $\left|\log _{2}(1 / Q(w))-r\right| \leqslant \Delta+1$, and for $1 \leqslant k \leqslant f-1$, $B_{k}=\left\{m^{\prime}:\left|\log _{2}\left(1 / Q\left(m^{\prime}\right)\right)-r\right| \leqslant(k+1) \Delta+1\right\}$. Finally, Bob finds a chain $\mathcal{A}^{\prime}$ of length $f$ and size at most $s$ such that $\mathcal{B} \in S^{1}\left(\mathcal{A}^{\prime}\right)$.

As $\mathcal{B} \in S^{1}(\mathcal{A})$ as well, we have that $S^{1}(\mathcal{A}) \cap S^{1}\left(\mathcal{A}^{\prime}\right) \neq \varnothing$. Hence, if the set of chains of length $f$ and size at most $s$ can be colored so that for chains $\mathcal{A}, \mathcal{A}^{\prime}$ in this set with $S^{1}(\mathcal{A}) \cap S^{1}\left(\mathcal{A}^{\prime}\right) \neq \varnothing$ and $A_{0} \neq A_{0}^{\prime}, \mathcal{A}$ and $\mathcal{A}^{\prime}$ receive different colors, then Bob can recover the original message $m$ if Alice transmits the color of $\mathcal{A}$ (along with the integers $s$ and $r$ ). In particular, Bob only needs to find a chain $\mathcal{A}^{\prime}$ as above that has the same color as $\mathcal{A}$, and then the single element of $A_{0}^{\prime}$ is guaranteed to be $m$ [5]. Therefore, to minimize the expected length of the encoding, our goal is to color the set of such chains with as few colors as possible subject to the coloring condition above. Recall from above that Haramaty and Sudan showed that the set of chains of length $2 k$ and size at most $s$ can be colored with at most $2^{6(s+1)} \cdot \log ^{(k)} N$ colors in this way. This leads to an expected length of $2^{\frac{H(P)}{\epsilon}+2 \Delta \log ^{*} N+O(1)}$ (for an error rate of at most $\epsilon$ ), which is not quite constant in $N$. In order to achieve an encoding of constant size, one possibility is to reduce the number of colors $2^{O(s)} \log ^{(k)} N$ to $O\left(\log ^{(k)} N\right)$ for all $k \leqslant \log ^{*} N-c$, for some absolute constant $c$. Using this upper bound for $k=\log ^{*} N-c$ immediately gives a constant number of colors, which corresponds to an encoding of constant size. Determining whether or not this is possible motivates our work.

\subsection{Overview of results}

In Section 2, we show that the upper bound of $2^{O(s)} \cdot \log ^{(k)} N$ on the chromatic number of $U(N, s, k)$ can be improved to $2^{O\left(2^{2 s}\right)} \cdot \log ^{(2 k)} N$. For any $\epsilon>0$, we can further improve the bound to $2^{O\left(2^{(1+\epsilon) s}\right)} \cdot \log ^{(2 k)} N$ for a certain subgraph of $U(N, s, k)$ whose vertices are chains $\left\langle\alpha, A_{1}, \ldots, A_{\delta}\right\rangle$ where $\left|A_{i}\right|, 1 \leqslant i \leqslant \delta$, grows exponentially with $i$. To obtain these upper bounds, we prove a result relating the chromatic numbers of graphs $G, H$ when there is a graph homomorphism $\phi: H \rightarrow G$ :

Theorem 1. If $\chi(G)>2, \phi: H \rightarrow G$ is a graph homomorphism, and $r \in \mathbb{N}$ such that $|\phi(N(v))| \leqslant r$ for each $v \in H$, then $\chi(H) \leqslant\left\lceil 2^{r} \log \log \chi(G)\right\rceil$.

Haramaty and Sudan [5] showed that in the context of Theorem 1, we have that $\chi(H) \leqslant 2 r(r+1) \log \chi(G)$. Thus Theorem 1 is an improvement when $\chi(G)$ is large com- 
pared to $r$. Given an arbitrary graph $G$, we may construct a graph $H$ and a homomorphism $\phi$ as in Theorem 1 as follows: we let $V(H)=\{\langle v, S\rangle\}_{v \in V(G), S \subset N(v) \cup\{v\}, v \in S,|S| \leqslant r+1}$, and a pair $(\langle v, S\rangle,\langle u, T\rangle) \in E(H)$ if and only if $v \in T$ and $u \in S$ and $u \neq v$. We will call $H$ the restricted neighborhood graph of $G$ and write $H=R N(G)$. Next, we define the homomorphism $\phi: R N(G) \rightarrow G$ that maps $\langle v, S\rangle$ to $v$. It is clear that $\phi$ is indeed a homomorphism, and moreover that $|\phi(N(\langle v, S\rangle))| \leqslant r$ for all $\langle v, S\rangle \in V(H)$, as each element of $\phi(N(\langle v, S\rangle))$ must be in $S-\{v\}$.

In Section 4, we prove a lower bound of $2^{\frac{s-2 k-4}{2}} \cdot \log (2 k) N+o(1)$ on the chromatic number of $U(N, s, k)$ as $N \rightarrow \infty$. To establish this bound, we prove a result that is similar in nature to Theorem 1, except it provides a lower bound on $\chi(H)$ in terms of $\chi(G)$. To state this result, we define a graph homomorphism $\phi: H \rightarrow G$ to be complete if it satisfies the following property: for any $x, z \in V(H)$, if $\phi(z) \in \phi(N(x))$ and $\phi(x) \in \phi(N(z))$, then $(x, z) \in E(H)$. Then we have:

Theorem 2. Suppose $\phi: H \rightarrow G$ is a complete graph homomorphism such that for any $w \in G$ and neighbors $u_{1}, \ldots, u_{r}$ of $w$, there is some $v \in V(H)$ such that $u_{1}, \ldots, u_{r} \in$ $\phi(N(v))$ and $\phi(v)=w$. Then if $\chi(H)=n$, we have that $\chi(G) \leqslant 2^{2 n+2^{n / 2^{r-2}}}$.

Together, Theorems 1 and 2 show that if $\phi: H \rightarrow G$ is a complete graph homomorphism and $r_{1}, r_{2} \in \mathbb{N}$ such that $|\phi(N(v))| \leqslant r_{1}$ for each $v \in V(H)$ and for any $w \in G$ and neighbors $u_{1}, \ldots, u_{r_{2}}$ of $w$, there is some $v \in V(H)$ such that $u_{1}, \ldots, u_{r_{2}} \in \phi(N(v))$ and $\phi(v)=w$, then

$$
2^{2^{\chi(H) / 2^{r_{1}}}} \leqslant \chi(G) \leqslant 2^{2 \chi(H)+2^{\chi(H) / 2^{r_{2}-2}}} .
$$

Note that we will always have $r_{1} \geqslant r_{2}$ in such a scenario.

Note that for a graph $G$, the homomorphism $\phi: R N(G) \rightarrow G$ as described above is complete: if $\langle v, S\rangle,\langle u, T\rangle \in V(R N(G))$, and $\phi(\langle v, S\rangle) \in \phi(N(\langle u, T\rangle))$ and $\phi(\langle u, T) \in$ $\phi(N(\langle v, S))$, then $v \in T$ and $u \in S$, but $u \neq v$. This clearly implies that $(\langle v, S\rangle,\langle u, T\rangle) \in$ $E(R N(G))$. Note also that for any $w \in G$ and neighbors $u_{1}, \ldots, u_{r}$, then the vertex $\left\langle w,\left\{u_{1}, \ldots, u_{r}, w\right\}\right\rangle$ has the property that $u_{1}, \ldots, u_{r} \in \phi\left(\left\langle w,\left\{u_{1}, \ldots, u_{r}, w\right\}\right\rangle\right)$ and $\phi\left(\left\langle w,\left\{u_{1}, \ldots, u_{r}, w\right\}\right\rangle\right)=w$. Therefore, with $H=R N(G)$, it follows from Theorems 1 and 2 that (1) holds with $r_{1}=r_{2}=r$.

\section{Graph Independence and Upper Bounds}

Many of the results presented in this section are generalizations of analogues proven by Erdős et al. in [3], and which were discovered independently in [12]. As such, we will make a change in notation and write $U(m, R, \delta)$ instead of $U(N, s, k)$ to be consistent with the notation of [3]. Erdös et al. considered the following question: we say that a graph $G$ has a local $(m, R)$-coloring $[3,8,10,11,12]$ if it has a proper coloring with $m$ colors that uses at most $T$ colors in the neighborhood of each vertex (including the vertex itself). Then given that $G$ has a local $(m, R)$-coloring, how can the chromatic number of $G$ be bounded above? Erdös et al. obtained nearly tight bounds on the answer to this question for values of $m, R$ in certain ranges. Many of the results presented below make progress towards 
answering the following generalization of this question, which Erdös et al. also stated ([3], Definitions 4.1 and 4.2), and which was also raised by Szegedy and Vishwanathan [12]: we say that $G$ has a local $(m, R, \delta)$-coloring if it has a proper coloring with $m$ colors that uses at most $R$ colors in the distance- $\delta$ neighborhood $N_{\delta}(v)$ of each vertex $v$. Then for $\delta>1$, by how much can the upper bound on the chromatic number of $G$ be improved (since if $G$ has a local $(m, R, \delta)$ coloring, then it certainly has a local $(m, R)$-coloring)?

For a graph $G$, we will consider in this section collections $\mathcal{F}_{G}$ of pairs $(v, S)$ that satisfy $v \in V(G), S \subset V(G)$, and for each $u \in S,(v, u) \in E(G)$. We will be particularly interested in such collections $\mathcal{F}_{G}$ that are defined as follows: for a graph $H$, and a graph homomorphism $\phi: H \rightarrow G$, we define the collection of tuples $\mathcal{F}_{G, \phi}$ as follows:

$$
\mathcal{F}_{G, \phi}=\{(\phi(v), \phi(N(v)))\}_{v \in H} .
$$

We first make the following definition pertaining to such collections $\mathcal{F}_{G}$, which generalizes Definition 4.5 (as well as Definition 1.4) in [3], as well as an analogous definition in [12]. In doing so, we identify the vertices in $V(G)$ with the integers $\{1,2, \ldots,|V(G)|\}$. Also, for a set $S \subset V(G)$, we let $\min S=\min _{u \in S} u$.

Definition 3. Consider a graph $G$, a collection $\mathcal{F}_{G}$ as above, and $n \in \mathbb{N}$. Then the system of sets $\left\{A_{u, v}\right\}_{1 \leqslant u<v \leqslant|V(G)|,(u, v) \in E} \subseteq \mathcal{P}([n])$ is $\left(G, n, \mathcal{F}_{G}\right)$-independent, if, for any $(v, S) \in \mathcal{F}_{G}$ with $v>\min S$,

$$
\bigcap_{u<v, u \in S,(u, v) \in E} A_{u, v}-\bigcup_{w>v, w \in S,(v, w) \in E} A_{v, w} \neq \varnothing,
$$

and for any $(v, S) \in \mathcal{F}_{G}$ with $v<\min S$, we have

$$
[n]-\bigcup_{w>v, w \in S,(v, w) \in E} A_{v, w} \neq \varnothing .
$$

Now we prove two lemmas which establish a link between the existence of $\left(G, n, \mathcal{F}_{G}\right)$ independent systems and the chromatic number of graphs, which generalize Lemma 4.4 (as well as Lemma 1.2) in [3].

Lemma 4. For a graph homomorphism $\phi: H \rightarrow G$, suppose that there is a $\left(G, n, \mathcal{F}_{G, \phi}\right)$ independent system. Then $\chi(H) \leqslant n$.

Proof. Let $\left\{A_{u, v}\right\}_{1 \leqslant u<v \leqslant|V(G)|,(u, v) \in E(G)}$ be a $\left(G, n, \mathcal{F}_{G, \phi}\right)$-independent system. Take any vertex $x \in V(H)$, and if $\phi(x)>\min \phi(N(x))$ and $N(x)$ is nonempty, define

$$
g(x)=\min \left\{\bigcap_{u \in \phi(N(x)), u<\phi(x)} A_{u, \phi(x)}-\bigcup_{v \in \phi(N(x)), v>\phi(x)} A_{\phi(x), v}\right\},
$$

where the set on the right hand side of the above equation is nonempty by $\left(G, n, \mathcal{F}_{G, \phi}\right)$ independence. If $\phi(x)<\min (\phi(N(x))$ or $N(x)$ is empty, then define

$$
g(x)=\min \left\{[n]-\bigcup_{v \in \phi(N(x)), v>\phi(x)} A_{\phi(x), v}\right\} .
$$


We claim that $g$ is a proper coloring of $H$. To see this, take 2 vertices $x, y \in V(H)$ with $(x, y) \in E(H)$, and suppose without loss of generality that $\phi(x)<\phi(y)$. The fact that $\phi(x) \in \phi(N(y))$ implies that $g(y) \in A_{\phi(x), \phi(y)}$. We also have that $\phi(y) \in \phi(N(x))$, so $g(x) \notin A_{\phi(x), \phi(y)}$, which implies that $g(x) \neq g(y)$, as desired.

Lemma 5. If $\chi(H) \leqslant n$, and $\phi: H \rightarrow G$ is a complete graph homomorphism, then there is a $\left(G, n, \mathcal{F}_{G, \phi}\right)$-independent system.

Proof. Suppose $g$ is a proper $n$-coloring of $H$. We define a $\left(G, n, \mathcal{F}_{G, \phi}\right)$-independent system $\left\{A_{u, v}\right\}$ as follows. For $(u, v) \in E(G)$, with $1 \leqslant u<v \leqslant|V(G)|$, we let

$$
A_{u, v}=\{g(x): x \in V(H), \phi(x)=v, u \in \phi(N(x))\} .
$$

We claim that this system is $\left(G, n, \mathcal{F}_{G, \phi}\right)$-independent. For suppose not; there are two possibilities:

1. There is some $x \in V(H), \phi(x)>\min \phi(N(x))$, such that

$$
\bigcap_{u \in \phi(N(x)), u<\phi(x)} A_{u, \phi(x)}-\bigcup_{v \in \phi(N(x)), v>\phi(x)} A_{\phi(x), v}=\varnothing .
$$

Let $\xi=g(x)$. We claim that

$$
\xi \in \bigcap_{u \in \phi(N(x)), u<\phi(x)} A_{u, \phi(x)}
$$

To see that this is the case, note that

$$
A_{u, \phi(x)}=\{g(y) \quad: \quad y \in V(H), \phi(y)=\phi(x), u \in \phi(N(y))\},
$$

so that for each $u \in \phi(N(x))$ with $u<\phi(x)$, we may simply choose $y=x$, and always have that $u \in \phi(N(y))$. Since $\phi(x)>\min \phi(N(x))$, there always exists at least one such $u$.

2. There is some $x \in V(H), \phi(x)<\min \phi(N(x))$, such that

$$
[n]-\bigcup_{v \in \phi(N(x)), v>\phi(x)} A_{\phi(x), v}=\varnothing .
$$

Again, let $\xi=g(x)$, so that $\xi \in[n]$.

In both cases above, there must exist $v>\phi(x)$, with $v \in \phi(N(x))$, such that $\xi \in$ $A_{\phi(x), v}$. In particular, this means that $\xi=g(z)$, for some $z \in V(H)$ with $\phi(z)=v$ and $\phi(x) \in \phi(N(z))$. Since $\phi$ is complete, this immediately implies that $(z, x) \in E(H)$, which is a contradiction to the fact that both $x$ and $z$ are colored $\xi$. This completes the proof.

Lemmas 4 and 5 immediately imply the following: 
Proposition 6. If $\phi: H \rightarrow G$ is a complete graph homomorphism, then $\chi(H) \leqslant n$ if and only if there exists a $\left(G, n, \mathcal{F}_{G, \phi}\right)$-independent system.

From Proposition 6, in order to prove upper bounds on the chromatic number of a graph $H$, we need to prove the existence of $\left(G, n, \mathcal{F}_{G, \phi}\right)$-independent systems for appropriate choices of $\phi, G$. To do so, we will use a result of Kleitman and Spencer [7] on the existence of families of independent sets.

Definition 7 (Kleitman and Spencer, [7]). If $S$ is an $n$-element set, then the $k$ subsets $A_{1}, \ldots, A_{k} \subseteq S$ are defined to be $k$-independent if all $2^{k}$ intersections $\cap_{j=1}^{n} B_{j}$ (where $B_{j}$ can be either $A_{j}$ or $\bar{A}_{j}$, and where $A_{j}$ and $\bar{A}_{j}$ do not both appear among the $B_{j}$ ), are nonempty. More generally, the $m$ subsets $A_{1}, \ldots, A_{m} \subseteq S$, for $m \geqslant k$, are $k$-independent if each $k$-element subset of $\left\{A_{1}, \ldots, A_{m}\right\}$ is $k$-independent.

Another way of stating the independence of $A_{1}, \ldots, A_{k}$ is that all $2^{k}$ portions of the Venn diagram relating $A_{1}, \ldots, A_{k}$, are nonempty. Kleitman and Spencer defined $f(n, k)$ to the the maximum size of a collection of a $k$-independent collection of subsets of an $n$-element set. Their main result was:

Theorem 8 (Kleitman and Spencer, [7]). We have:

$$
f(n, 2)=\left(\begin{array}{c}
n-1 \\
\lfloor n / 2\rfloor-1
\end{array}\right)
$$

and there are absolute constants $d_{1} \geqslant 1, d_{2}$ so that for each fixed $k \geqslant 3$, there is a sufficiently large $N_{k}$, so that for all $n \geqslant N_{k}$,

$$
2^{d_{1} n 2^{-k} / k} \leqslant f(n, k) \leqslant 2^{d_{2} n 2^{-k}} .
$$

The proof of the lower bound for $f(n, k)$ in [7] was probabilistic, but an explicit construction was later found in [1]. We now use the existence of independent collections of sets as guaranteed to exist in Theorem 8 to prove the existence of $\left(G, n, \mathcal{F}_{G}\right)$-independent systems for appropriate choices of $G, \mathcal{F}_{G}$ in Lemma 9 below. The proof of this lemma is similar to that of Theorem 2.4 in [3].

Lemma 9. If there is an $r$-independent collection of $k$ subsets of an $n$-element set, and $G$ is a graph with $\chi(G)=h \leqslant 2^{k}$, and $\mathcal{F}_{G}$ is a collection of pairs $(v, S)$ (with $v \in V(G)$, $S \subset V(G)$ and $S$ only contains neighbors of $v)$ where each such pair has $|S| \leqslant r$, then there is a $\left(G, n, \mathcal{F}_{G}\right)$ independent system.

Proof. Suppose $\chi: G \rightarrow[h]$ is a proper coloring of $G$, and by re-ordering the vertices of $G$ we can assume without loss of generality that $\chi$ respects the ordering of $V$; that is, for $u<v \in V(G)$, we have that $\chi(u) \leqslant \chi(v)$.

Next suppose that we have an $n$-element set $Q$, and subsets $A_{1}, \ldots, A_{k} \subseteq Q$ that are $r$-independent, for some $r \leqslant k$. Moreover recall that $h \leqslant 2^{k}$. Let $\mathscr{C}=\left\{A_{1}, \ldots, A_{k}\right\}$, and 
suppose that $\left\{Y_{i}: 1 \leqslant i \leqslant 2^{k}\right\}$ is an enumeration of the power set $\mathcal{P}(\mathscr{C})$ with $\left|Y_{i}\right| \leqslant\left|Y_{j}\right|$ for $i<j$. Define the system of subsets

$$
\mathcal{T}=\left\{A_{u, v}: 1 \leqslant u<v \leqslant|V(G)|,(u, v) \in E(G)\right\}
$$

by letting $A_{u, v} \in Y_{\chi(v)}-Y_{\chi(u)}$, where we have used the fact that $\chi(v)>\chi(u)$ for $v>u$ such that $(u, v) \in E$ (so in particular, we cannot have that $\chi(u)=\chi(v)$ ). We are also using the fact here that each $\chi(u) \leqslant 2^{k}$, which follows from $h \leqslant 2^{k}$. We claim that the collection $\mathcal{T}$ is $\left(G, n, \mathcal{F}_{G}\right)$-independent as long as for each $(v, S) \in \mathcal{F}_{G}$, we have $|S| \leqslant r$. To see this, note that for any $(v, S) \in \mathcal{F}_{G}$, we have that

$$
\bigcap_{u<v, u \in S,(u, v) \in E} A_{u, v}-\bigcup_{w>v, w \in S,(w, v) \in E} A_{v, w}=A_{q_{1}} \cap \cdots \cap A_{q_{s}} \cap \bar{A}_{p_{1}} \cap \cdots \cap \bar{A}_{p_{t}},
$$

for $1 \leqslant q_{1}, \ldots, q_{s}, p_{1}, \ldots, p_{t} \leqslant k$, and $s+t \leqslant|S| \leqslant r$. (If $v<\min S$, then the relevant quantity is $\bar{A}_{p_{1}} \cap \cdots \cap \bar{A}_{p_{t}}$, and $s=0$.) Note that each of $A_{q_{1}}, \ldots, A_{q_{s}}$ are equal to one of $A_{u, v}$, and that each of $A_{p_{1}}, \ldots, A_{p_{t}}$ are equal to one of $A_{v, w}$. For any $A_{u, v}$, we have that $A_{u, v} \in Y_{\chi(v)}$, and for any $A_{v, w}$ we have that $A_{v, w} \notin Y_{\chi(v)}$. Therefore, we have that $A_{u, v} \neq A_{v, w}$ for all valid choice of $u, w$. Therefore, by $r$-independence of the collection $\mathscr{C}=\left\{A_{1}, \ldots, A_{k}\right\}$, we have that $(2)$ is nonempty. This implies that $\mathcal{T}$ is $\left(G, n, \mathcal{F}_{G}\right)$ independent.

Theorem 1 now follows as an immediate consequence of Lemma 9 and Theorem 8:

Proof of Theorem 1. Given $\phi: H \rightarrow G$ with $|\phi(N(v))| \leqslant r$ for each $v \in H$, note that the collection $\mathcal{F}_{G, \phi}$ satisfies $|S| \leqslant r$ for each $(v, S) \in \mathcal{F}_{G, \phi}$. Next, by Theorem 8 , there is an $r$-independent collection of $2^{n 2^{-r} / r}$ subsets of an $n$-element set. Therefore, by Lemma 9 , as long as $\chi(G) \leqslant 2^{2^{n 2^{-r} / r}}$, we have that a $\left(G, n, \mathcal{F}_{G, \phi}\right)$-independent system exists. Lemma 4 then implies that $\chi(H) \leqslant n$. Note that $\chi(G) \leqslant 2^{2^{n 2^{-r} / r}}$ is equivalent to $n \geqslant r 2^{r} \log \log \chi(G)$, which implies that $\chi(H) \leqslant\left\lceil r 2^{r} \log \log \chi(G)\right\rceil$.

\subsection{Chain graphs: basic facts}

Before deriving our upper bounds on $\chi(U(m, R, \delta))$ for various choices of $m, R, \delta$, we first establish some basic facts about the chain graphs $U(m, R, \delta)$. The first result, Proposition 10 below, explains how the result of Haramaty and Sudan [5] implies an upper bound on $\chi(U(m, R, \delta)$ ) (which is weaker than ours). Haramaty and Sudan [5] showed that for $m, R, \delta \in \mathbb{N}$, the set $V(U(m, R, 2 \delta))$ can be colored with at most $2^{6(R+1)} \log ^{(\delta)} m$ colors, such that for any two chains $\mathcal{A}=\left\langle\alpha, \ldots, A_{2 \delta}\right\rangle, \mathcal{B}=\left\langle\beta, \ldots, B_{2 \delta}\right\rangle$ in this set, if $S^{1}(\mathcal{A}) \cap S^{1}(\mathcal{B}) \neq \varnothing$ and $\alpha \neq \beta$, then $\mathcal{A}$ and $\mathcal{B}$ are colored by different colors. By Proposition 10, this implies that there is a proper vertex coloring of the graph $U(m, R, \delta)$ with at most $2^{(6(R+1))} \cdot \log ^{(\delta)} m$ colors.

Proposition 10. Let $R, \delta, c \in \mathbb{N}$. Then the following two statements are equivalent: 
1. There exists a c-coloring of the set of all chains in Chain $(m)$ that have size at most $s$ and length $2 \delta$ such that for any two chains $\mathcal{A}=\left\langle\alpha, \ldots, A_{2 \delta}\right\rangle, \mathcal{B}=\left\langle\beta, \ldots, B_{2 \delta}\right\rangle$ in this set, if $S^{1}(\mathcal{A}) \cap S^{1}(\mathcal{B}) \neq \varnothing$ and $\alpha \neq \beta$, then $\mathcal{A}$ and $\mathcal{B}$ are colored by different colors.

2. There is a proper c-coloring of $U(m, R, \delta)$.

Proof. We first suppose that (1) is true, and construct a proper $c$-coloring of $U(m, R, \delta)$. In particular, for any vertex, say $\mathcal{A}=\left\langle\alpha, A_{1}, \ldots, A_{\delta}\right\rangle$, we may give it the color of $\left\langle\alpha, A_{1}, A_{1}, A_{2}, A_{2}, \ldots, A_{\delta}, A_{\delta}\right\rangle$, which is a chain of length $2 \delta$ and size at most $R$. To see that this is a proper coloring, consider an edge $\left(\left\langle\alpha, A_{1}, \ldots, A_{\delta}\right\rangle,\left\langle\beta, B_{1}, \ldots, B_{\delta}\right\rangle\right)$ of $U(m, R, \delta)$. Then

$$
\begin{aligned}
& \left\langle\alpha,\{\alpha\} \cup\{\beta\}, A_{1} \cup B_{1}, A_{1} \cup B_{1}, \ldots, A_{\delta-1} \cup B_{\delta-1}, A_{\delta-1} \cup B_{\delta-1}\right\rangle \\
\in & S^{1}\left(\left\langle\alpha, A_{1}, A_{1}, A_{2}, A_{2}, \ldots, A_{\delta}, A_{\delta}\right\rangle\right) \cap S^{1}\left(\left\langle\beta, B_{1}, B_{1}, B_{2}, B_{2}, \ldots, B_{\delta}, B_{\delta}\right\rangle\right) .
\end{aligned}
$$

Since also $\alpha \neq \beta$, by the definition of the coloring in statement (1), it follows that $\left\langle\alpha, A_{1}, \ldots, A_{\delta}\right\rangle$ and $\left\langle\beta, B_{1}, \ldots, B_{\delta}\right\rangle$ receive different colors.

Next suppose that we are given a proper $c$-coloring of $U(m, R, \delta)$. For each chain $\mathcal{A}=$ $\left\langle\alpha, A_{1}, \ldots, A_{2 \delta}\right\rangle$ of size at most $R$ and length $2 \delta$, we give $\mathcal{A}$ the color of $\left\langle\alpha, A_{2}, A_{4}, \ldots, A_{2 \delta}\right\rangle$ $\in V(U(m, R, \delta))$. To see that this coloring satisfies the condition in (1), suppose that for chains $\mathcal{A}=\left\langle\alpha, A_{1}, \ldots, A_{2 \delta}\right\rangle$ and $\mathcal{B}=\left\langle\beta, B_{1}, \ldots, B_{2 \delta}\right\rangle$ of length $2 \delta$ and size at most $R$, the chain $\mathcal{C}=\left\langle\gamma, C_{1}, \ldots, C_{2 \delta-1}\right\rangle \in S^{1}(\mathcal{A}) \cap S^{1}(\mathcal{B})$ and $\alpha \neq \beta$. Then $\alpha \in C_{1} \subseteq B_{2}$, $\beta \in C_{1} \subseteq A_{2}$, and for $1 \leqslant i \leqslant \delta-1, A_{2 i} \subseteq C_{2 i+1} \subseteq B_{2(i+1)}$ and $B_{2 i} \subseteq C_{2 i+1} \subseteq A_{2(i+1)}$. This implies that $\left\langle\alpha, A_{2}, \ldots, A_{2 \delta}\right\rangle$ and $\left\langle\beta, B_{2}, \ldots, B_{2 \delta}\right\rangle$ are adjacent in the graph $U(m, R, \delta)$, which implies that $\mathcal{A}$ and $\mathcal{B}$ indeed receive different colors this way.

Our goal is to determine if it is possible to obtain some kind of bound on $\chi(U(m, R, \delta))$ that improves the bound $\chi(U(m, R, \delta)) \leqslant 2^{O(R)} \cdot \log ^{(\delta)} m$ from [5]. To do so, we will use Theorems 1 and 2 to reason about $\chi(U(m, R, \delta))$; first, though, we must establish an appropriate graph homomorphism $\phi$ used in those theorems.

We define the map $\phi: V(U(m, R, \delta)) \rightarrow V(U(m, R, \delta-1))$ by

$$
\phi\left(\left\langle\alpha, A_{1}, \ldots, A_{\delta}\right\rangle\right)=\left\langle\alpha, A_{1}, \ldots, A_{\delta-1}\right\rangle .
$$

It is immediate that $\phi$ is a graph homomorphism. It is also complete:

Lemma 11. For any choice of $m, R, \delta$, the graph homomorphism $\phi$ defined in (3) is complete.

Proof. Consider any chains $\mathcal{A}, \mathcal{B} \in V(W(m, \sigma, \delta))$, and suppose that $\phi(\mathcal{A}) \in \phi(N(\mathcal{B}))$ and $\phi(\mathcal{B}) \in \phi(N(\mathcal{A}))$. Let us write $\mathcal{A}=\left\langle\alpha, A_{1}, \ldots, A_{\delta}\right\rangle$ and $\mathcal{B}=\left\langle\beta, B_{1}, \ldots, B_{\delta}\right\rangle$. Since $\phi(\mathcal{A}) \in \phi(N(\mathcal{B}))$, we have that for some $\mathcal{C}=\left\langle\gamma, C_{1}, \ldots, C_{\delta}\right\rangle \in N(\mathcal{B}), \phi(\mathcal{A})=\phi(\mathcal{C})$. In particular, this means that $\alpha=\gamma$ and $A_{i}=C_{i}$ for $1 \leqslant i \leqslant \delta-1$. Since $\mathcal{C} \in N(\mathcal{B})$, it follows that $\alpha \in B_{1}, \beta \in A_{1}$, and for $1 \leqslant i \leqslant \delta-2, A_{i} \subseteq B_{i+1}$ and $B_{i} \subseteq A_{i+1}$. This also gives us that $A_{\delta-1} \subseteq B_{\delta}$. In a symmetric manner, since $\phi(\mathcal{B}) \in \phi(N(\mathcal{A}))$, we have that $B_{\delta-1} \subseteq A_{\delta}$. This implies that $(\mathcal{A}, \mathcal{B}) \in E(W(m, \sigma, \delta))$. 
The next lemma states that completeness respects restrictions to induced subgraphs. It will be useful when we prove lower bounds on the chromatic number of induced subgraphs of $U(m, R, \delta)$ in Section 4. Given a graph homomorphism $\phi: H \rightarrow G$ and an induced subgraph $H^{\prime}$ of $H$, we will denote the restriction of $\phi$ to $H^{\prime}$ by $\phi_{H^{\prime}}: H^{\prime} \rightarrow G$. Moreover, in the proof of the below lemma, for a vertex $x \in V(H)$, we denote by $N_{H}(x)$ the neighborhood of $x$ in $H$ and by $N_{H^{\prime}}(x)$ the neighborhood of $x$ in $H^{\prime}$.

Lemma 12. Suppose that $G, H$ are graphs and $H^{\prime}$ is an induced subgraph of $H$. Suppose that $\phi: H \rightarrow G$ is a complete graph homomorphism. Then the restriction $\phi_{H^{\prime}}: H^{\prime} \rightarrow G$ is also complete.

Proof. Consider vertices $x, z \in V\left(H^{\prime}\right)$ such that $\phi_{H^{\prime}}(x) \in \phi_{H^{\prime}}\left(N_{H^{\prime}}(z)\right)$ and $\phi_{H^{\prime}}(z) \in$ $\phi_{H^{\prime}}\left(N_{H^{\prime}}(x)\right)$. Our aim is to show that $(x, z) \in E\left(H^{\prime}\right)$. Since $N_{H^{\prime}}(x) \subseteq N_{H}(x)$ and $N_{H^{\prime}}(z) \subseteq N_{H}(z)$, it follows that $\phi(x) \in \phi\left(N_{H}(z)\right)$ and $\phi(z) \in \phi\left(N_{H}(x)\right)$. Since $\phi$ is complete, we have $(x, z) \in E(H)$ as a consequence. Since $H^{\prime}$ is an induced subgraph and $(x, z) \in E\left(H^{\prime}\right)$, it follows that $(x, z) \in E\left(H^{\prime}\right)$, as desired.

\section{Upper bound on the chromatic number of chain graphs}

Now we use the results in the previous section to derive an upper bound on the chromatic number of $U(m, R, \delta)$, as well as an improved upper bound on a subgraph of $U(m, R, \delta)$ whose vertices are chains that grow exponentially in size (here recall that $m, R, \delta \in \mathbb{N}$, where $m$ denotes the size of the universe, $R$ denotes that maximum size of the chains, and $\delta$ denotes the length of the chains). We begin with a small lemma that allows us to bound the " $r$ " parameter in Theorem 1.

Lemma 13. For $\mathcal{A} \in V(U(m, R, \delta))$, we have that $|\phi(N(\mathcal{A}))| \leqslant 2^{2 \cdot s z(\mathcal{A})} \leqslant 2^{2 R}$.

Proof. Write $\mathcal{A}=\left\langle\alpha, A_{1}, \ldots, A_{\delta}\right\rangle$, and consider any $\left\langle\beta, B_{1}, \ldots, B_{\delta}\right\rangle \in \phi(N(\mathcal{A}))$. We must have $\beta \in A_{1}, \alpha \in B_{1}, B_{1} \subseteq A_{2}$, and $A_{i-1} \subseteq B_{i} \subseteq A_{i+1}$ for $2 \leqslant i \leqslant \delta-1$, so the number of choices for $\left\langle\beta, B_{1}, \ldots, B_{\delta-1}\right\rangle$ is at most

$$
\begin{aligned}
\left|A_{1}\right| \cdot 2^{\left|A_{2}\right|-1} \cdot 2^{\left|A_{3}\right|-\left|A_{1}\right|} \cdot 2^{\left|A_{4}\right|-\left|A_{2}\right|} \cdots 2^{\left|A_{\delta}\right|-\left|A_{\delta-2}\right|} & \leqslant 2^{\left|A_{1}\right|+\cdots+\left|A_{\delta}\right|-\left(\left|A_{1}\right|+\left|A_{2}\right|+\cdots+\left|A_{\delta-2}\right|\right)} \\
& \leqslant 2^{2 \cdot\left|A_{\delta}\right|}=2^{2 \cdot s z(\mathcal{A})} .
\end{aligned}
$$

When $R \ll m$, Theorem 14 improves the bound $\chi(U(m, R, \delta)) \leqslant 2^{O(R)} \cdot \log ^{(\delta)} m$ of $[5]$ to $\chi(U(m, R, \delta)) \leqslant 2^{O\left(2^{2 R}\right)} \cdot \log ^{(2 \delta)} m$.

Theorem 14. If $\log { }^{(2 \delta-2)} m \geqslant 2^{2^{2+2 R}}$, then $\chi(U(m, R, \delta)) \leqslant 2^{2^{2+2 R}} \cdot \log ^{(2 \delta)} m$.

Proof. We use induction on $\delta$. For the base case $\delta=0$, we have that $U(m, R, \delta)=K_{m}$, the complete graph on $m$ vertices. Then $\chi\left(K_{m}\right)=m=\log ^{(0)} m$.

Now suppose the result is true for $\delta-1$. Let $G=U(m, R, \delta-1)$, so that $\chi(G) \leqslant$ $2^{2^{2+2 R}} \cdot \log ^{(2 \delta-2)} m$. Let $H=U(m, R, \delta)$. Consider the graph homomorphism $\phi: H \rightarrow G$ defined in (3), which is complete by Lemma 11 . Now consider any $(v, S) \in \mathcal{F}_{G, \phi}$ (recall 
that $\mathcal{F}_{G, \phi}$ is the set of all pairs $(\phi(\mathcal{A}), \phi(N(\mathcal{A})))$, where $\left.\mathcal{A} \in V(U(m, R, \delta))\right)$. By Lemma 13, we have that $|S| \leqslant 2^{2 R}$. By Theorem 1, we have that

$$
\chi(U(m, R, \delta)) \leqslant\left\lceil 2^{2 R} 2^{2^{2 R}} \log \log \chi(U(m, R, \delta-1))\right\rceil .
$$

Since $2^{2^{2+2 R}} \leqslant \log ^{(2 \delta-2)} m$, the above equation implies that

$$
\chi(U(m, R, \delta)) \leqslant\left\lceil 2^{2 R} 2^{2^{2 R}}\left(1+\log ^{(2 \delta)} m\right)\right] \leqslant 2^{2+2 R} 2^{2^{2 R}} \log { }^{(2 \delta)} m \leqslant 2^{2^{2+2 R}} \log ^{(2 \delta)} m .
$$

We will occasionally write the upper bound in Theorem 14 as $\chi(U(m, R, \delta)) \leqslant$ $2^{2^{2+2 R}} \log ^{(2 \delta)} m+o(1)$ (as $m \rightarrow \infty$ ) since for fixed $R, \delta$, the upper bound holds for sufficiently large $m$.

Next we explain how to improve the upper exponent in the upper bound of Theorem 14 from $2+2 R$ to to $2+R \cdot \frac{r}{r-1}$ for a particular subgraph of $U(m, R, \delta)$ defined by a parameter $r$. In particular, given $r, m, \sigma, \delta \in \mathbb{N}$ with $r \geqslant 2$, we define the graph $W_{r}(m, \sigma, \delta)$ as follows:

$$
V=\left\{\left\langle\alpha, A_{1}, \ldots, A_{\delta}\right\rangle: \alpha \in A_{1} \subset A_{2} \subset \cdots \subset A_{\delta} \subset[m], \quad \forall 1 \leqslant i \leqslant \delta,\left|A_{i}\right|=r^{i-1} \cdot r^{\sigma}\right\},
$$

and

$$
\begin{aligned}
E= & \left\{\left(\left\langle\alpha, A_{1}, \ldots, A_{\delta}\right\rangle,\left\langle\beta, B_{1}, \ldots, B_{\delta}\right\rangle\right):\right. \\
& \left.\alpha \neq \beta, \alpha \in B_{1}, \beta \in A_{1}, \quad \forall 1 \leqslant i \leqslant \delta-1: A_{i} \subseteq B_{i+1}, B_{i} \subseteq A_{i+1}\right\} .
\end{aligned}
$$

We refer the reader to Table 1 for a summary of the chain graphs considered in this paper, including $W_{r}(m, \sigma, \delta)$. Note that for any vertex (chain) $\mathcal{A} \in V\left(W_{r}(m, \sigma, \delta)\right)$, we have that $s z(\mathcal{A})=r^{\sigma+\delta-1}$, so $W_{r}(m, \sigma, \delta)$ is an induced subgraph of $U\left(m, r^{\sigma+\delta-1}, \delta\right)$. Therefore, by Theorem 14, we have that $\chi\left(W_{r}(m, \sigma, \delta)\right) \leqslant 2^{2^{2+2 r^{\sigma+\delta-1}}} \cdot \log ^{(2 \delta)} m$ for sufficiently large $m$. In Theorem 15, we prove that we can improve the upper bound to $2^{2^{2+\frac{r+1}{r} \cdot r} \sigma+\delta-1}$.

Theorem 15. For $r, \sigma, \delta, m \in \mathbb{N}$ with $r \geqslant 2$ and $\log ^{(2 \delta)} m \geqslant 1$, we have $\chi\left(W_{r}(m, \sigma, \delta)\right) \leqslant$ $2^{2^{2+r^{\sigma+\delta-2}(r+1)}} \cdot \log { }^{(2 \delta)} m$.

The proof of Theorem 15 is very similar to that of Theorem 14, except it uses the exponential growth of the chains in $V\left(W_{r}(m, \sigma, \delta)\right)$, and can be found in Appendix A.

\section{Lower bounds}

In this section we prove lower bounds on the chromatic number of $U(m, R, \delta)$ by establishing lower bounds on the chromatic number of certain induced subgraphs of $U(m, R, \delta)$. The key ingredient to doing so is Theorem 2, which we prove first. We state it below in a slightly different form:

Theorem 16. Suppose $\phi: H \rightarrow G$ is a complete graph homomorphism such that for any $w \in G$ and neighbors $u_{1}, \ldots, u_{r}$ of $w$, there is some $(w, S) \in \mathcal{F}_{G, \phi}$ such that $u_{1}, \ldots, u_{r} \in S$. Then if $\chi(H) \leqslant n$, we have that $\chi(G) \leqslant 2^{2 n+2^{n / 2^{r-2}}}$. 


\begin{tabular}{|c|c|c|c|}
\hline Graph & $\begin{array}{c}\text { Vertex growth } \\
\text { condition }\end{array}$ & $\chi$ upper bound & $\chi$ lower bound \\
\hline$U(m, R, \delta)$ & $\left|A_{\delta}\right| \leqslant R$ & $2^{2^{2+2 R} \log ^{(2 \delta)} m+o(1)}$ & $2^{\frac{R-2 \delta-4}{2}} \log ^{(2 \delta)} m+o(1)$ \\
\hline$W_{r}(m, \sigma, \delta)$ & $\begin{array}{c}\left|A_{i}\right|=r^{\sigma+i-1} \\
1 \leqslant i \leqslant \delta\end{array}$ & $2^{2^{2+r^{\sigma+\delta-2}(r+1)} \log ^{(2 \delta)} m}$ & $2^{\frac{r^{\sigma+\delta-2}(r-1)-5}{2}} \log ^{(2 \delta)} m+$ \\
\hline$Y(m, \delta)$ & $\begin{array}{c}\left.\left|A_{i}\right|=2 i+1\right) \\
1 \leqslant i \leqslant \delta\end{array}$ & $2^{2^{2+2(2 \delta+1)} \log ^{(2 \delta)} m}$ & $\log ^{(2 \delta)} m$ \\
\hline$Z(m, R, \delta)$ & $\begin{array}{c}\left|A_{i}\right|=2 i+1, \\
\left|A_{\delta}\right|=R-1,\end{array}$ & $2^{2^{2+2 R}} \log ^{(2 \delta)} m+o(1)$ & $2^{\frac{R-2 \delta-4}{2}} \log ^{(2 \delta)} m+o(1)$ \\
\hline
\end{tabular}

Table 1: Summary of chain graphs considered in this paper. In the second column ("Vertex growth condition") a typical vertex of any of these graphs is denoted by $\left\langle\alpha, A_{1}, \ldots, A_{\delta}\right\rangle$, with $\alpha \in A_{1} \subseteq \cdots \subseteq A_{\delta} \subseteq[m]$. In some cells, the best known known upper or lower bounds are determined by subgraph relations (for instance, $Y(m, \delta)$ is a subgraph of $U(m, 2 \delta+1, \delta)$, so $\chi(Y(m, \delta)) \leqslant \chi(U(m, 2 \delta+1, \delta)))$.

The proof of Theorem 16 (equivalently, Theorem 2) is similar to the proof of Theorem 2.3 in [3]. The bulk of this proof is contained in Lemma 17.

Lemma 17. Suppose $G$ is a graph and $\mathcal{F}_{G}$ is a collection of pairs $(v, S)$ (where $v \in V(G)$ and $S \subset V(G)$ ) such that for any $v \in G$ and neighbors $u_{1}, \ldots, u_{r}$ of $v$, there is some $(v, S) \in \mathcal{F}_{G, \phi}$ such that $u_{1}, \ldots, u_{r} \in S$. Also suppose that a $\left(G, n, \mathcal{F}_{G}\right)$-independent system exists. Then there is a partition of $V(G)$ into $n^{\prime}+1 \leqslant 2^{n-1}+1$ sets, say $T_{1}, T_{2}, \ldots, T_{n^{\prime}}, T_{n^{\prime}+1}$, and collections $\mathcal{F}_{G\left[T_{1}\right]}, \ldots, \mathcal{F}_{G\left[T_{n^{\prime}}\right]}$ such that:

1. $T_{n^{\prime}+1}$ is an independent set in $G$.

2. For $1 \leqslant i \leqslant n^{\prime}$, there is a $\left(G\left[T_{i}\right],\lfloor n / 2\rfloor, \mathcal{F}_{G\left[T_{i}\right]}\right)$-independent system.

3. For each $1 \leqslant i \leqslant n^{\prime}$, for any $v \in T_{i}$, and neighbors $u_{1}, \ldots, u_{r-1}$ in $G\left[T_{i}\right]$, there is some $(v, S) \in \mathcal{F}_{G\left[T_{i}\right]}$ such that $u_{1}, \ldots, u_{r-1} \in S$.

Proof. Associating $V(G)$ with $\{1, \ldots,|V(G)|\}$, let us denote a $\left(G, n, \mathcal{F}_{G}\right)$-independent system by $\left\{A_{u, v}: 1 \leqslant u<v \leqslant|V(G)|, u, v \in V(G)\right\}$. For $v \in V(G)$, and $A \subset[n]$ with $|A| \leqslant\lfloor n / 2\rfloor$, define $v$ to be of type $A$ if one of the statements below holds:

1. There exists $u<v$ with $A_{u, v}=A$, or

2. There exists $w>v$ with $A_{v, w}=[n]-A$.

Note that for any vertices $u<v$ with $(u, v) \in E(G)$, either $v$ is of type $A_{u, v}$ or $u$ is of type $[n]-A_{u, v}$, where the former holds if $\left|A_{u, v}\right| \leqslant\lfloor n / 2\rfloor$, and the latter holds if $\left|A_{u, v}\right| \geqslant\lceil n / 2\rceil$. Therefore, if $v$ is not of type $A$ for any $A$, then each of its neighbors is of type $A$ for some $A$, meaning that the set of vertices that are not of type $A$ for any $A$ form an independent 
set in $G$; let this set be $T_{n^{\prime}+1}$. The number of sets $A \subset[n]$ with $|A| \leqslant\lfloor n / 2\rfloor$ is at most $2^{n-1}$, meaning that if we index such sets by $A_{1}, A_{2}, \ldots, A_{n^{\prime}}$ with $n^{\prime} \leqslant 2^{n-1}$ we may let $T_{i}$ be the set of all vertices of type $A_{i}$ (if a vertex is of type $A$ for more than 1 set $A$, we pick $A$ arbitrarily).

We next define the collections $\mathcal{F}_{G\left[T_{i}\right]}$ as follows. Consider any pair $(v, S) \in \mathcal{F}_{G}$ that has the property that $v \in T_{i}$ for some $i$. There now is either some $u_{v}<v$ such that $A_{u_{v}, v}=A_{i}$ or some $u_{v}>v$ such that $A_{v, u_{v}}=[n]-A_{i}$. We will now include the pair $\left(v, S \cap T_{i}\right)$ in $\mathcal{F}_{G\left[T_{i}\right]}$ if and only if $u_{v} \in S$. In other words, we have

$$
\mathcal{F}_{G\left[T_{i}\right]}=\left\{\left(v, S \cap T_{i}\right):(v, S) \in \mathcal{F}_{G}, v \in T_{i}, u_{v} \in S\right\} .
$$

Next, for $1 \leqslant i \leqslant n^{\prime}$, consider the collection $\mathcal{F}_{G\left[T_{i}\right]}$, and pick any $v \in T_{i}$. Now consider any neighbors $u_{1}, \ldots, u_{r-1}$ of $v$ in $G\left[T_{i}\right]$. Since $v$ and $u_{v}$ are neighbors, we know that there exists some $(v, S) \in \mathcal{F}_{G}$ such that $u_{1}, \ldots, u_{r-1}, u_{v} \in S$. Therefore, letting $\hat{S}=S \cap T_{i}$, we have that $(v, \hat{S}) \in \mathcal{F}_{G\left[T_{i}\right]}$ and that $u_{1}, \ldots, u_{r-1} \in \hat{S}$.

Finally, we claim that for each $1 \leqslant i \leqslant n^{\prime}$ the system

$$
\left\{A_{u, v} \cap A_{i} \quad: \quad 1 \leqslant u<v \leqslant|V(G)|, u \in T_{i}, v \in T_{i}\right\}
$$

is $\left(G\left[T_{i}\right],\lfloor n / 2\rfloor, \mathcal{F}_{G\left[T_{i}\right]}\right)$-independent. To see this consider any $(v, S) \in \mathcal{F}_{G\left[T_{i}\right]}$. We wish to show that

$$
\left(\bigcap_{u<v, u \in S,(u, v) \in E\left(G\left[T_{i}\right]\right)} A_{u, v} \cap A_{i}\right)-\left(\bigcup_{w>v, w \in S,(v, w) \in E\left(G\left[T_{i}\right]\right)} A_{v, w} \cap A_{i}\right) \neq \varnothing
$$

if $v>\min S$, and that

$$
A_{i}-\left(\bigcup_{w>v, w \in S,(v, w) \in E\left(G\left[T_{i}\right]\right)} A_{v, w} \cap A_{i}\right) \neq \varnothing
$$

otherwise. We must consider two cases:

1. There is $u<v$ such that $A_{u, v}=A_{i}$. By construction of $\mathcal{F}_{G\left[T_{i}\right]}$, there is some $S^{\prime}$ with $S \cup\{u\} \subset S^{\prime} \operatorname{such}$ that $\left(v, S^{\prime}\right) \in \mathcal{F}_{G}$. If $v>\min S$, then (5) is satisfied by $\left(G, n, \mathcal{F}_{G}\right)$-independence of $\left\{A_{u, v}\right\}$, as $A_{i}$ will be one of the terms in the intersection in the definition of $\left(G, n, \mathcal{F}_{G}\right)$-independence and $v>u \geqslant \min S^{\prime}$. If $v<\min S$, then (6) is satisfied since $u<v$ and thus $A_{i}$ is again one term in the intersection in the definition of $\left(G, n, \mathcal{F}_{G}\right)$-independence.

2. There is $w>v$ such that $A_{v, w}=[n]-A_{i}$. By construction of $\mathcal{F}_{G\left[T_{i}\right]}$, there is some $S^{\prime}$ with $S \cup\{w\} \subset S^{\prime}$ such that $\left(v, S^{\prime}\right) \in \mathcal{F}_{G}$. If $v>\min S$, then $v>\min S^{\prime}$ as well, so (5) is satisfied by $\left(G, n, \mathcal{F}_{G}\right)$-independence of $\left\{A_{u, v}\right\}$, as $[n]-A_{i}$ will be one of the terms in the union in the definition of $\left(G, n, \mathcal{F}_{G}\right)$-independence. If $v<\min S$, then regardless of whether $v<\min S^{\prime},(6)$ is satisfied since again $[n]-A_{i}$ is one of the terms in the union in the definition of $\left(G, n, \mathcal{F}_{G}\right)$-independence. 
We have shown that each of (1), (2), (3) in the statement of the lemma hold.

Now we prove Theorem 16 .

Proof. Let us associate $V(G)$ with the set $\{1,2, \ldots,|V(G)|\}$. By Proposition 6 we have that there is a $\left(G, n, \mathcal{F}_{G, \phi}\right)$-independent system.

We now use induction on $r$ and $n$ to prove the following claim:

Claim 18. Suppose there exists a $\left(G, n, \mathcal{F}_{G}\right)$-independent system such that for any set of $r$ neighbors $\left\{u_{1}, \ldots, u_{r}\right\}$ of any vertex $v \in V(G)$, there is some $(v, S) \in \mathcal{F}_{G}$ with $\left\{u_{1}, \ldots, u_{r}\right\} \subseteq S$. Then $\chi(G) \leqslant 2^{2 n+2^{n / 2^{r-2}}}$.

We first prove the base case $r=2$. Let a $\left(G, n, \mathcal{F}_{G, \phi}\right)$-independent system be denoted $\left\{A_{u, v}\right\}_{1 \leqslant u<v \leqslant|V(G)|}$. We now color $G$ by giving $v \in V(G)$ the set $B_{v}:=\left\{A_{u, v}\right\}_{u \in N(v), u<v}$ as the color of $v$. In particular, the color of $v$ is a set of sets, each of which is a subset of $[n]$, meaning that the total number of colors is $2^{2^{n}} \leqslant 2^{2 n+2^{n / 2^{2-2}}}$. We claim that this gives a proper coloring of $G$. To see this, consider any edge $(v, w) \in E(G)$, such that $v<w$. If there is no $u<v$ with $(u, v) \in E(G)$, then $B_{v}$ is the empty set, whereas $B_{w}$ at least contains $A_{v, w}$, so certainly $B_{v} \neq B_{w}$. Also, note that for any $u<v$ with $(u, v) \in E(G)$, we have that $(v,\{u, w\}) \in \mathcal{F}_{G}$, meaning that $A_{u, v}-A_{v, w} \neq \varnothing$. Therefore, $A_{v, w}$ does not contain any element in $B_{v}$ as a subset, so in particular $B_{v}$ cannot contain $A_{v, w}$. However, $B_{w}$ does contain $A_{v, w}$, meaning that $B_{v}$ and $B_{w}$ are distinct, as desired.

Now assume that for all $r<r_{0}$ and $n<n_{0}$, Claim 18 holds. Suppose that there is a $\left(G, n_{0}, \mathcal{F}_{G}\right)$-independent system, where $\mathcal{F}_{G}$ is such that for any set of $r_{0}$ neighbors $\left\{u_{1}, \ldots, u_{r_{0}}\right\}$ of any vertex $v$, there is some $(v, S) \in \mathcal{F}_{G}$ with $\left\{u_{1}, \ldots, u_{r_{0}}\right\} \subseteq S$. By Lemma 17, we may partition $V(G)$ into sets $T_{1}, \ldots, T_{n^{\prime}}, T_{n^{\prime}+1}$, with $n^{\prime} \leqslant 2^{n_{0}-1}$, and such that properties $(1)-(3)$ in the lemma are satisfied. By the inductive hypothesis and conditions (2) and (3) of Lemma 17, we have that for $1 \leqslant i \leqslant n^{\prime}$,

$$
\chi\left(G\left[T_{i}\right]\right) \leqslant 2^{n_{0}+2^{\left\lfloor\frac{n_{0}}{2}\right\rfloor / 2^{r_{0}-3}}} \leqslant 2^{n_{0}+2^{n_{0} / 2^{r_{0}-2}}} .
$$

By condition (1) in Lemma 17, we have that $\chi\left(G\left[T_{n^{\prime}+1}\right]\right)=1$. Therefore, we may color the vertices of $G$ by the product coloring of the unique $i$ such that any $v \in T_{i}$ and the coloring of $G\left[T_{i}\right]$, which gives:

$$
\chi(G) \leqslant 1+n^{\prime} \cdot 2^{n_{0}+2^{n_{0} / 2^{r_{0}-2}}} \leqslant 2^{2 n_{0}+2^{n_{0} / 2^{r_{0}-2}}},
$$

where we have also used that only one color is needed for $T_{n^{\prime}+1}$. This gives the desired result.

For any fixed $r$, the function $n \mapsto 2^{2 n+2^{n / 2^{r-2}}}$ is a strictly increasing continuous function of $n$ for $n \in \mathbb{R}^{+}$, so for any $m \in \mathbb{R}^{+}$with $m \geqslant 2$, there is a unique $n$ with $m=2^{2 n+2^{n / 2^{r-2}}}$. Therefore, we may define $P_{r}(m)$ to be the inverse of the function $n \mapsto 2^{2 n+2^{n / 2^{r-2}}}$ for $n \in \mathbb{R}^{+}$. The next two lemmas establish an asymptotic form for $P_{r}(m)$ which will be useful in applying Theorem 16. 
We let $\mathcal{W}: \mathbb{R}^{+} \rightarrow \mathbb{R}^{+}$be the Lambert $\mathcal{W}$-function, defined as the inverse of the function $f(x)=x e^{x}$. (In particular, we are letting $\mathcal{W}$ denote the principal branch of the Lambert $\mathcal{W}$-function, restricted to the positive reals.) Then we have the following:

Lemma 19. If $m=2^{2 n+2^{n / 2^{r-2}}}$, then

$$
n=\frac{2 \ln m-2^{r} \mathcal{W}\left(2^{1-r} m^{2^{1-r}} \ln 2\right)}{4 \ln 2} .
$$

Proof. Define $\hat{n}=\frac{2 \ln m-2^{r} \mathcal{W}\left(2^{1-r} m^{2^{1-r}} \ln 2\right)}{4 \ln 2}$. Our goal is to show that $\hat{n}=n$. Note that

$$
2^{\hat{n}}=\exp \left(\frac{\ln m}{2}-2^{r-2} \mathcal{W}\left(2^{1-r} m^{2^{1-r}} \ln 2\right)\right)=m^{1 / 2} \cdot \exp \left(-2^{r-2} \mathcal{W}\left(2^{1-r} m^{2^{1-r}} \ln 2\right)\right),
$$

which implies

$$
2^{\hat{n} / 2^{r-2}}=m^{1 / 2^{r-1}} \cdot \exp \left(-\mathcal{W}\left(2^{1-r} m^{2^{1-r}} \ln 2\right)\right)
$$

and

Next we claim that

$$
2 \hat{n}=\log _{2} m-\frac{2^{r-1}}{\ln 2} \cdot \mathcal{W}\left(2^{1-r} m^{2^{1-r}} \ln 2\right) .
$$

$$
m^{1 / 2^{r-1}} \cdot \exp \left(-\mathcal{W}\left(2^{1-r} m^{2^{1-r}} \ln 2\right)\right)=\frac{2^{r-1}}{\ln 2} \cdot \mathcal{W}\left(2^{1-r} m^{2^{1-r}} \ln 2\right),
$$

which, by definition of $\mathcal{W}$, is equivalent to

$$
\frac{m^{2^{1-r}} \cdot \ln 2}{2^{r-1}}=2^{1-r} \cdot m^{2^{1-r}} \cdot \ln 2,
$$

which is trivially true. By (7), (8), and (9), $2^{\hat{n} / 2^{r-2}}=\log _{2} m-2 \hat{n}$, so $m=2^{2 \hat{n}+2^{\hat{n} / 2^{r-2}}}$. Since the function $n \mapsto 2^{2 n+2^{n / 2^{r-2}}}$ is a strictly increasing function of $n$ for any $r$, it must be the case that $\hat{n}=n$, as desired.

In the below lemma, recall that log denotes the base-2 logarithm while ln denotes the natural logarithm.

Lemma 20. For a fixed $r$, we have that $P_{r}(m)=2^{r-2} \cdot \log \log m+o(1)$ as $m \rightarrow \infty$.

Proof. By Lemma 19, we have $P_{r}(m)=\frac{2 \ln m-2^{r} \mathcal{W}\left(2^{1-r} m^{2^{1-r}} \ln 2\right)}{4 \ln 2}$. It was shown in [2] that $\mathcal{W}(x)=\ln x-\ln \ln x+o(1)$ as $x \rightarrow \infty$. For fixed $r$, note that $2^{1-r} m^{2^{1-r}} \ln 2 \rightarrow \infty$ as $m \rightarrow \infty$. Therefore,

$$
\begin{aligned}
4 \ln 2 \cdot P_{r}(m)= & 2 \ln m-2^{r}\left(\ln \left(2^{1-r} m^{2^{1-r}} \ln 2\right)-\ln \ln \left(2^{1-r} m^{2^{1-r}} \ln 2\right)+o(1)\right) \\
= & 2 \ln m-2^{r}\left((1-r) \ln 2+2^{1-r} \ln m+\ln \ln 2\right. \\
& \left.-\ln \left((1-r) \ln 2+2^{1-r} \ln m+\ln \ln 2\right)+o(1)\right) \\
= & 2^{r}(r-1) \ln 2-2^{r} \ln \ln 2+o(1)+2^{r} \ln \left((1-r) \ln 2+2^{1-r} \ln m+\ln \ln 2\right) \\
= & 2^{r}(r-1) \ln 2-2^{r} \ln \ln 2+2^{r}(1-r) \ln 2+2^{r} \ln \ln m+o(1) \\
= & 2^{r} \ln \ln m-2^{r} \ln \ln 2+o(1) \\
= & 2^{r} \ln \log m+o(1) .
\end{aligned}
$$


To arrive at equality (10) we have used that $\ln \left(2^{1-r} \ln m+(1-r) \ln 2+\ln \ln 2\right)=$ $\ln \left(2^{1-r} \ln m\right)+o(1)$ as $m \rightarrow \infty$. From the above chain of equalities we then get that $P_{r}(m)=2^{r-2} \frac{\ln \log m}{\ln 2}+o(1)=2^{r-2} \log \log m+o(1)$, as desired.

Using the previous results we next derive a lower bound on the chromatic number of $U(m, R, \delta)$ as well as of the subgraphs $W_{r}(m, \sigma, \delta)$ considered in the previous section. To do this we define two more families of graphs, denoted by $Y(m, \delta)$ and $Z(m, R, \delta)$ (see also Table 1). Vertices of the graph $Y(m, \delta)$ are chains that grow arithmetically in size; in particular for $m, \delta \in \mathbb{N}$, we define $Y(m, \delta)$ to be the subgraph of $U(m, 2 \delta+1, \delta)$ induced by the set of vertices:

$$
V(Y(m, \delta)):=\left\{\left\langle\alpha, A_{1}, \ldots, A_{\delta}\right\rangle: \alpha \in A_{1} \subset \cdots \subset A_{\delta} \subset[m], \forall 1 \leqslant i \leqslant \delta,\left|A_{i}\right|=2 i+1\right\} .
$$

Next, if moreover $R \geqslant 2 \delta+1$ we define $Z(m, R, \delta)$ to be the subgraph of $U(m, R, \delta)$ induced by the set of vertices:

$$
V(Z(m, R, \delta))=\left\{\left\langle\alpha, A_{1}, \ldots, A_{\delta}\right\rangle: \begin{array}{c}
\alpha \in A_{1} \subset \cdots \subset A_{\delta} \subset[m],\left|A_{\delta}\right|=R, \\
\forall 1 \leqslant i \leqslant \delta-1,\left|A_{i}\right|=2 i+1
\end{array}\right\} .
$$

By restriction to $Z(m, R, \delta),(3)$ defines a graph homomorphism $\phi: Z(m, R, \delta) \rightarrow$ $Y(m, \delta-1)$. By Lemmas 11 and $12, \phi$ is complete.

We will derive a lower bound on $\chi(Z(m, R, \delta))$, which will then imply lower bounds on $\chi(U(m, R, \delta))$ and $\chi\left(W_{r}(m, \sigma, \delta)\right)$, for appropriate choices of $r, \sigma$. We will need a few basic facts to do so. The following theorem gives a lower bound on the chromatic number of $Y(m, \delta)$ :

Theorem 21 ([9], Theorem 2.1). For all $m, \delta$ with $\log ^{(2 \delta)} m \geqslant 1, \chi(Y(m, \delta)) \geqslant \log ^{(2 \delta)} m$.

Essentially equivalent statements of the above theorem can also be found in Theorem 2.7 of [5] and Theorem 7 of [4].

The next lemma states a key property of the map $\phi: Z(m, R, \delta) \rightarrow Y(m, \delta-1)$ that allows us to apply Theorem 16.

Lemma 22. For any $m, R, \delta$ with $R \geqslant 2 \delta+1$, the homomorphism $\phi: Z(m, R, \delta) \rightarrow$ $Y(m, \delta-1)$ satisfies the following property for $r \in \mathbb{N}$ with $r \leqslant \frac{R-(2 \delta-1)}{2}$. For any chains $\mathcal{A}, \mathcal{B}^{(1)}, \ldots, \mathcal{B}^{(r)} \in V(Y(m, \delta-1))$, such that $\left(\mathcal{A}, \mathcal{B}^{(i)}\right) \in E(Y(m, \delta-1))$ for $1 \leqslant i \leqslant r$, there exists a pair $(\mathcal{A}, S) \in \mathcal{F}_{Y(m, \delta-1), \phi}$ such that for $1 \leqslant i \leqslant r, \mathcal{B}^{(i)} \in S$.

Proof. Let us write $\mathcal{A}=\left\langle\alpha, A_{1}, \ldots, A_{\delta-1}\right\rangle$ and $\mathcal{B}^{(i)}=\left\langle\beta^{(i)}, B_{1}^{(i)}, \ldots, B_{\delta-1}^{(i)}\right\rangle$ for $1 \leqslant i \leqslant r$. Since $B_{\delta-2}^{(i)} \subset A_{\delta-1}$ for each $i$, we have that $\left|B_{\delta-1}^{(i)} \backslash A_{\delta-1}\right| \leqslant 2$. Define

$$
A_{\delta}=A_{\delta-1} \cup \bigcup_{1 \leqslant i \leqslant r} B_{\delta-1}^{(i)} \backslash A_{\delta-1}
$$

so that $\left|A_{\delta}\right| \leqslant 2 \delta-1+2 r \leqslant R$. If necessary, add a few arbitrary elements of $[m]$ to $A_{\delta}$ so that its size is exactly $R$. Now define $\tilde{\mathcal{A}}:=\left\langle\alpha, A_{1}, \ldots, A_{\delta-1}, A_{\delta}\right\rangle \in V(Z(m, R, \delta))$. Now indeed each $\mathcal{B}^{(i)} \in \phi(N(\tilde{\mathcal{A}}))$, so we may take $S=\phi(N(\tilde{\mathcal{A}}))$, completing the proof. 
We may now derive a lower bound on $\chi(Z(m, R, \delta))$ :

Theorem 23. We have $\chi(Z(m, R, \delta))>2^{\frac{R-2 \delta-4}{2}} \log ^{(2 \delta)} m+o(1)$ as $m \rightarrow \infty$.

Proof. By Theorem 21, we have that $\chi(Y(m, \delta)) \geqslant \log ^{(2 \delta-2)} m$. Let us write $n=$ $\chi(Z(m, R, \delta))$. By Theorem 16 with $r=\left\lfloor\frac{R-(2 \delta-1)}{2}\right\rfloor$ and Lemma 22, we have that $\log ^{(2 \delta-2)} m \leqslant \chi(Y(m, \delta)) \leqslant 2^{2 n+2^{n / 2^{r-2}}}$. By Lemma 20, we get that

$$
\chi(Z(m, R, \delta)) \geqslant 2^{\left\lfloor\frac{R-2 \delta+1}{2}\right\rfloor-2} \cdot \log ^{(2 \delta)} m+o(1),
$$

as $m \rightarrow \infty$, as desired.

Theorem 23 implies the following: given $m, R, \delta$, there exists a graph $G$ (namely, $Z(m, R, \delta))$ with a proper coloring with $m$ colors such that there are at most $R$ colors in the distance- $\delta$ neighborhood of each vertex of $G$, such that $\chi(G)>2^{\frac{R-2 \delta-4}{2}} \cdot \log ^{(2 \delta)} m+o(1)$. This generalizes Theorem 2.3 in [3] and Theorem 6 in [12].

Since $Z(m, R, \delta)$ is a subgraph of $U(m, R, \delta)$, the following corollary of Theorem 23 is immediate:

Corollary 24. We have $\chi(U(m, R, \delta))>2^{\frac{R-2 \delta-4}{2}} \log ^{(2 \delta)} m+o(1)$ as $m \rightarrow \infty$.

We next embed $Z(m / 2, R, \delta)$ in $W_{r}(m, \sigma, \delta)$ for appropriate choices of $R, \sigma, r$, allowing us to derive a lower bound on $\chi\left(W_{r}(m, \sigma, \delta)\right)$ in the below corollary.

Corollary 25. For $m, r, \sigma, \delta \in \mathbb{N}$ with $r, \sigma \geqslant 2$, we have

$$
\chi\left(W_{r}(m, \sigma, \delta)\right)>2^{\frac{r^{\sigma+\delta-2}(r-1)-5}{2}} \log ^{(2 \delta)} m+o(1)
$$

as $m \rightarrow \infty$.

Proof. Fix $r, \sigma, \delta$. Let $\tilde{r}=r^{\sigma+\delta-1}-r^{\sigma+\delta-2}$. For sufficiently large $m$, we define a graph homomorphism $\psi: Z(m / 2,2 \delta-1+\tilde{r}, \delta) \rightarrow W_{r}(m, \sigma, \delta)$, as follows. First choose sets $C_{1} \subseteq C_{2} \subseteq \cdots \subseteq C_{\delta-1} \subseteq\{m / 2+1, \ldots, m\}$ such that $\left|C_{i}\right|=r^{\sigma+i-1}-(2 i+1)$ for $1 \leqslant i \leqslant \delta-1$. This is certainly possible for large enough $m$.

Now consider a chain $\mathcal{A}=\left\langle\alpha, A_{1}, \ldots, A_{\delta}\right\rangle \in V(Z(m / 2,2 \delta-1+\tilde{r}, \delta))$. We map it to the chain $\psi(\mathcal{A}):=\left\langle\alpha, A_{1} \cup C_{1}, A_{2} \cup C_{2}, \ldots, A_{\delta-1} \cup C_{\delta-1}, A_{\delta} \cup C_{\delta-1}\right\rangle$. From this construction, as well as the fact that $\left|A_{\delta} \cup C_{\delta-1}\right|=\left|A_{\delta-1}\right|+\left|C_{\delta-1}\right|+\left|A_{\delta} \backslash A_{\delta-1}\right|=r^{\sigma+\delta-2}+\tilde{r}=r^{\sigma+\delta-1}$, we have that $\psi(\mathcal{A}) \in V\left(W_{r}(m, \sigma, \delta)\right)$. It is also evident from the construction that $\psi$ is indeed a graph homomorphism, meaning that $\chi(Z(m / 2,2 \delta-1+\tilde{r}, \delta)) \leqslant \chi\left(W_{r}(m, \sigma, \delta)\right)$. By Theorem 23, it follows that

$$
\chi\left(W_{r}(m, \sigma, \delta)\right) \geqslant 2^{\frac{\tilde{r}-5}{2}} \log ^{(2 \delta)}(m / 2)+o(1) \geqslant 2^{\frac{r^{\sigma+\delta-2}(r-1)-5}{2}} \log ^{(2 \delta)} m+o(1),
$$

as desired. 


\section{Conclusion}

Now we compare the necessary conditions needed on the homomorphism $\phi: H \rightarrow G$ to obtain lower (Theorem 16) and upper (Lemma 9) bounds on $\chi(H)$ if we already know $\chi(G)$. We assume that $\phi$ is complete. The requirement in Theorem 16 is that for any $v \in G$ and neighbors $u_{1}, \ldots, u_{r} \in V(G)$ of $v$, there is some $(v, S) \in \mathcal{F}_{G, \phi}$ such that $u_{1}, \ldots, u_{r} \in S$. The requirement in Lemma 9 is that for any $(v, S) \in \mathcal{F}_{G}$, we have that $|S| \leqslant r$. Note that in the specific case where $G=K_{m}$ and $H=U(m, r+1,1)$, these conditions are equivalent. However, for other values of $G$ (in particular, say $G=Z(m, R, \delta)$ ), we will have that for any $(v, S) \in \mathcal{F}_{G, \phi}$, the size of $S$ is much larger than the maximum number of neighbors $u_{1}, \ldots, u_{r}$ of $v$ for which there is some $(v, S) \in \mathcal{F}_{G, \phi}$ such that $u_{1}, \ldots, u_{r} \in S$. The difference between these two conditions creates the gap in the bound

$$
2^{\frac{r^{\sigma+\delta-2}(r-1)-5}{2}} \log ^{(2 \delta)} m+o_{m}(1) \leqslant \chi\left(W_{r}(m, \sigma, \delta)\right) \leqslant 2^{2^{2+r^{\sigma+\delta-2}(r+1)}} \cdot \log ^{(2 \delta)} m
$$

on $\chi\left(W_{r}(m, \sigma, \delta)\right)$, from Theorem 15 and Corollary 25, as well as the gap in the bound

$$
2^{\frac{R-2 \delta-4}{2}} \log (2 \delta) m+o_{m}(1) \leqslant \chi(U(m, R, \delta)) \leqslant 2^{2^{2+2 R}} \log ^{(2 \delta)} m+o_{m}(1),
$$

from Theorem 14 and Corollary 24. We remark that prior to our work the best known lower bound on $\chi\left(W_{r}(m, \sigma, \delta)\right)$ and $\chi(U(m, R, \delta))$ was $\log ^{(2 \delta)} m$, though it is still of great interest to close the gap in (11) and (12).

The lower bound of $2^{\frac{R-2 \delta-4}{2}} \log ^{(2 \delta)} m+o(1)$ on $\chi(U(m, R, \delta))$ from Theorem 23 casts doubt on the possibility that there is a constant-length encryption scheme with no error as described in Section 1. Recall that improving the upper bound on $\chi(U(m, R, \delta))$ from

[5] by replacing the term $2^{O(R)}$ by a constant (independent of $R$ ) is sufficient for finding such a compression scheme. We have shown that it is not possible to do this for small values of $\delta$, whereas previously such a result was only known for the case $\delta=1[3,12]$.

\section{Acknowledgements}

I am grateful to Madhu Sudan for calling my attention to this problem and for helpful discussions, to Aaron Potechin for helpful suggestions and for pointing out a few minor errors in the proofs, and to an anonymous referee for helpful suggestions. 


\section{A Proof of Theorem 15}

Lemma 26 uses the exponential growth of the chains $\mathcal{A} \in V\left(W_{r}(m, \sigma, \delta)\right)$ to obtain a better upper bound on $|\phi(N(\mathcal{A}))|$ than the one in Lemma 13.

Lemma 26. For $\mathcal{A} \in V\left(W_{r}(m, \sigma, \delta)\right)$, we have that $|\phi(N(\mathcal{A}))| \leqslant 2^{r^{\sigma+\delta-2}(r-1)}$.

Proof. Exactly as in Lemma 13, using the fact that for all $\left\langle\beta, B_{1}, \ldots, B_{\delta-1}\right\rangle \in \phi(N(\mathcal{A}))$, we must have $\beta \in A_{1}, \alpha \in B_{1}, B_{1} \subseteq A_{2}$, and $A_{i-1} \subseteq B_{i} \subseteq A_{i+1}$ for $2 \leqslant i \leqslant \delta-1$, the number of elements in $\phi(N(\mathcal{A}))$ is at most

$$
2^{\left|A_{1}\right|+\cdots+\left|A_{\delta}\right|-\left(\left|A_{1}\right|+\cdots+\left|A_{\delta-2}\right|\right)}=2^{r^{\sigma+\delta-2}(1+r)} .
$$

Note that the bound in Lemma 26 can be written as $|\phi(N(\mathcal{A}))| \leqslant 2^{s z(\mathcal{A}) \cdot \frac{r+1}{r}}$ which is better than the bound $|\phi(N(\mathcal{A}))| \leqslant 2^{2 \cdot s z(\mathcal{A})}$ from Lemma 13.

Proof of Theorem 15. We use induction on $\delta$. For the base case $\delta=0$, we have that $W_{r}(m, \sigma, \delta)=K_{m}$, the complete graph on $m$ vertices. Then clearly $\chi\left(K_{m}\right)=m=$ $\log ^{(0)} m$.

Now suppose the result is true for $\delta-1$. Let $G=W_{r}(m, \sigma, \delta-1)$, so that $\chi(G) \leqslant$ $2^{2^{2+r^{\sigma+\delta-2}(r+1)}} \cdot \log { }^{(2 \delta-2)} m$. Let $H=W_{r}(m, \sigma, \delta)$. Next, (3) defines a graph homomorphism $\phi: H \rightarrow G$, by restricting to the induced subgraph $H=W_{r}(m, \sigma, \delta)$ of $U\left(m, r^{\sigma+\delta-1}, \delta\right)$. By Lemma 26, for each pair $(v, S) \in \mathcal{F}_{G, \phi}$, we have that $|S| \leqslant 2^{r^{\sigma+\delta-2}(r+1)}$.

Let $\rho=2^{r^{\sigma+\delta-2}(r+1)}$. By Theorem 1, we have that

$$
\chi\left(W_{r}(m, \sigma, \delta)\right) \leqslant\left\lceil\rho 2^{\rho} \cdot \log \log \chi\left(W_{r}(m, \sigma, \delta-1)\right)\right\rceil .
$$

If $2^{2^{2+r^{\sigma+\delta-3}(r+1)}} \leqslant \log ^{(2 \delta-2)} m$, then the above equation implies that

$$
\begin{aligned}
\chi\left(W_{r}(m, \sigma, \delta)\right) & \leqslant\left\lceil\rho 2^{\rho}\left(1+\log ^{(2 \delta)} m\right)\right\rceil \\
& \leqslant 2^{2+r^{\sigma+\delta-2}(r+1)} \cdot 2^{2^{r^{\sigma+\delta-2}(r+1)}} \cdot \log ^{(2 \delta)} m \\
& \leqslant 2^{2^{2+r^{\sigma+\delta-2}(r+1)}} \cdot \log ^{(2 \delta)} m .
\end{aligned}
$$

If $2^{2^{2+r^{\sigma+\delta-3}(r+1)}}>\log ^{(2 \delta-2)} m$, then since the function $\frac{\log \log n}{n}$ is a decreasing function of integers $n$ for $n \geqslant 4$, we have that

$$
\frac{\log ^{(2 \delta)} m}{\log ^{(2 \delta-2)} m}>\frac{2+r^{\sigma+\delta-3}(r+1)}{2^{2^{2+r^{\sigma+\delta-3}(r+1)}}},
$$

which implies that

$$
\frac{\log ^{(2 \delta)} m \cdot 2^{2^{2+r^{\sigma+\delta-2}(r+1)}}}{\log ^{(2 \delta-2)} m \cdot 2^{2^{2+r^{\sigma+\delta-3}(r+1)}}} \geqslant \frac{\left(2+r^{\sigma+\delta-3}(r+1)\right) \cdot 2^{2^{2+r^{\sigma+\delta-2}(r+1)}}}{\left(2^{2^{2+r^{\sigma+\delta-3}(r+1)}}\right)^{2}}>1,
$$

where the last inequality follows from $2+r^{\sigma+\delta-2}(r+1) \geqslant 3+r^{\sigma+\delta-3}(r+1)$, so we may use the trivial bound $\chi\left(W_{r}(m, \sigma, \delta)\right) \leqslant \chi\left(W_{r}(m, \sigma, \delta-1)\right) \leqslant \log ^{(2 \delta-2)} m \cdot 2^{2^{2+r^{\sigma+\delta-3}(r+1)}} \leqslant 2^{2^{2+r^{\sigma+\delta-2}(r+1)}} \cdot \log ^{(2 \delta)} m$. 


\section{References}

[1] Noga Alon. Explicit construction of exponential sized families of k-independent sets. Discrete Mathematics, 58(2):191-193, 1986.

[2] Rob Corless, Gaston Gonnet, D. E. G. Hare, David Jeffrey, and Donald Knuth. On the Lambert W Function. Advances in Computational Mathematics, 5(1):329-359, December 1996.

[3] Paul Erdős, Zoltán Füredi, András Hajnal, Péter Komjáth, Vojtěch Rödl, and Ákos Seress. Coloring graphs with locally few colors. Discrete Mathematics, 59(1):21-34, April 1986.

[4] Paul Erdős and András Hajnal. Some remarks on set theory. IX: Combinatorial problems in measure theory and set theory. Michigan Math Journal, 11(2):107-127, 1964.

[5] Elad Haramaty and Madhu Sudan. Deterministic Compression with Uncertain Priors. Algorithmica, 76(3):630-653, November 2016.

[6] Brendan Juba, Adam T. Kalai, Sanjeev Khanna, and Madhu Sudan. Compression without a common prior: an information-theoretic justification for ambiguity in language. In Innovations in Computer Science, pages 79-86, 2011.

[7] Daniel J. Kleitman and Joel Spencer. Families of k-independent sets. Discrete Mathematics, 6(3):255-262, January 1973.

[8] János Körner, Concetta Pilotto, and Gábor Simonyi. Local chromatic number and Sperner capacity. Journal of Combinatorial Theory, Series B, 95(1):101-117, September 2005 .

[9] Nathan Linial. Locality in distributed graph algorithms. SIAM Journal on Computing, 21(1):193-201, 1992.

[10] Gábor Simonyi and Gábor Tardos. Local chromatic number, Ky Fan's theorem, and circular colorings. Combinatorica, 26(5):587-626, 2006.

[11] Gábor Simonyi and Gábor Tardos. On directed local chromatic number, shift graphs, and Borsuk-like graphs. Journal of Graph Theory, 66(1):65-82, 2010.

[12] Márió Szegedy and Sundar Vishwanathan. Locality Based Graph Coloring. In Proceedings of the Twenty-fifth Annual ACM Symposium on Theory of Computing, STOC '93, pages 201-207, New York, NY, USA, 1993. ACM. 